\title{
Estudio Comparativo de Crecimiento Estaturo Ponderal y Acceso a Lactancia Materna Durante el Primer Año de Vida de Niños con Fisura Labio Máxilo Palatina versus Niños sin Fisura
}

\author{
Comparative Growth and Development Study in Children with \\ Cleft Lip Palate Fissure versus Children without Fissure
}

Cordero Erita ${ }^{1,2}$; Priscilla Madrid ${ }^{3}$; Espinoza Iris ${ }^{4}$; Consuelo Ulloa ${ }^{1,5,6} \&$ Pantoja Roberto ${ }^{1}$

CORDERO E.; MADRID, P.; ESPINOZA, I.; ULLOA, C. \& PANTOJA R. Estudio comparativo de crecimiento estaturo ponderal y acceso a lactancia materna durante el primer año de vida de niños con fisura labio máxilo palatina versus niños sin fisura. Int. J. Odontostomat., 14(1):35-41, 2020.

RESUMEN: La fisura labio máxilo palatina (FLMP) es un problema de salud pública en Chile. Consiste en la falta de fusión del labio y/o los procesos palatinos, generando un desequilibrio músculo esqueletal que impide el desarrollo facial normal. Afectando algunas funciones vitales como respiración, succión y deglución pudiendo ocasionar problemas nutricionales. Evaluar el crecimiento estaturo ponderal y acceso a lactancia materna de niños con fisura labio máxilo palatina (FLMP) comparados con niños sin fisura durante el primer año de vida. Estudio de tipo observacional, analítico de cohorte retrospectivo a partir de los datos provenientes de entrevistas a las madres de niños con FLMP no sindrómica atendidos durante el año 2017 y niños sin FLMP perteneciente a la misma región. En niño/as sin FLMP predomina la lactancia materna exclusiva y en niños/as con FLMP lactancia mixta $(47,7 \%)$ y uso exclusivo de leche de fórmula (33.3 \%). Los niños/ as con FLMP durante el primer año de vida pesan en promedio $0,45 \mathrm{Kg}$ y miden $1,11 \mathrm{~cm}$ menos que los niños/as sin fisura. Los niños/as con FLMP reciben menos lactancia materna y tienen un menor crecimiento estaturo ponderal que los niños/sin FLMP durante el primer año de vida, siendo el peso el parámetro de crecimiento más afectado.

PALABRAS CLAVE: fisura labio palatina, lactancia materna, peso, talla.

\section{INTRODUCCIÓN}

La fisura labio máxilo palatina (FLMP) es un problema de salud pública en Chile. Consiste en la falta de fusión del labio y/o los procesos palatinos, generando un desequilibrio músculo esqueletal que impide el desarrollo facial normal (Markus et al., 1992). Cauvi \& Leiva (2004) describen una clasificación anatómica que reconoce cuatro tipos: fisura de labio ( $F L)$, fisura de paladar $(F P)$, fisura labiopalatina (FLP) y fisuras atípicas (FA).

En Chile, según el Estudio Colaborativo Latino Americano de Malformaciones Congénitas (ECLAMC), la incidencia promedio entre el 2001 y 2010, en los hospitales participantes del estudio, para FP fue de 7,0 por $10.000 \mathrm{RN}$ vivos y FL con o sin compromiso palatino de 14,0 por $10.000 \mathrm{RN}$ vivos (Ministerio de Salud, 2015). La etiología es multifactorial, incluye: genética, edad materna, consumo de fármacos, tabaco y alcohol, antecedentes familiares, ocupación, nivel educacional y lugar de residencia de la madre (Gilda-Silva-Lopes et al., 2013).

Algunas funciones como respiración, succión y

\footnotetext{
${ }^{1}$ Servicio de Cirugía Oral y Maxilofacial, Hospital Clínico San Borja Arriarán. Santiago de Chile.

${ }^{2}$ Departamento de Cirugía Oral y Máxilofacial, Facultad de Odontología, Universidad de Chile, Santiago, Chile.

${ }^{3}$ Cirujano Dentista, Universidad de Chile, Santiago, Chile.

${ }^{4}$ Departamento de Patología y Medicina Oral, Centro de Epidemiología y Vigilancia de las Enfermedades Orales (CEVEO), Facultad de Odontología, Universidad de Chile, Santiago, Chile.

${ }^{5}$ Fonoaudióloga, Universidad de Chile, Santiago, Chile.

${ }^{6}$ CESFAM Las Mercedes, Servicio de Salud Metropolitano Central, Santiago, Chile.
} 
CORDERO E.; MADRID, P.; ESPINOZA, I.; ULLOA, C. \& PANTOJA R. Estudio comparativo de crecimiento estaturo ponderal y acceso a lactancia materna durante el primer año de vida de niños con fisura labio máxilo palatina versus niños sin fisura. Int. J. Odontostomat., 14(1):35-41, 2020.

deglución se ven alteradas debido a la comunicación entre la cavidad nasal y la cavidad oral. El recién nacido puede presentar complicaciones funcionales ocasionando problemas nutricionales y baja ganancia de peso (Cortés Araya et al., 2002; Reid, 2004; Gil-daSilva-Lopes et al.).

La alimentación debe ser adecuada con el fin de promover aporte calórico suficiente parar favorecer el crecimiento de los niños con FLMP debido a que necesitan alcanzar un peso y condiciones de salud ideales para ser sometidos a cirugías correctivas (Amstalden-Mendes \& Gil-da-Silva-Lopes, 2006). De acuerdo a la cronología funcional de Delaire, utilizada en el Hospital San Borja de Arriarán (HSBA) los pacientes deben ser intervenidos a los 6 meses con 8 kilos de peso para la anestesia general y recuperación (Cortés Araya et al.). Se recomienda promover la lactancia ya que se asocia a una mayor ganancia de peso (Ministerio de Salud).

\section{MATERIAL Y MÉTODO}

Es un estudio de tipo observacional, analítico de cohorte retrospectivo. Se incluyeron niños y niñas con FLMP no sindrómica, atendidos en el Servicio de Cirugía Maxilofacial del HSBA de la ciudad de Santiago entre el 1 de Enero y 31 de Diciembre 2017, nacidos entre enero 2013 y diciembre 2016. El grupo de estudio final se completó en 30 niños con FLMP no sindrómica.

El grupo control fueron niños(as) sin FLMP u otras anomalías congénitas, que asistieron programa de Control de Niño Sano entre el mes de enero y marzo 2018 en el Centro de Salud Familiar Las Mercedes de la ciudad de Santiago. Se seleccionaron 30 pacientes consecutivos que tuvieran entre 1 y 3 años, con registros de peso, talla y circunferencia craneal desde el nacimiento hasta el año de vida.

En ambos grupos se excluyeron niños/as que hubiesen nacido antes de las 36 semanas de gestación, que nacieran en embarazos múltiples y cuyas madres hubiesen presentado patología del embarazo y/o no firmaran el consentimiento informado.

Se contactó a las madres, padres o tutores de los niños/as de ambos grupos, de forma presencial o telefónica. Durante la entrevista se aplicó a la madre un cuestionario respecto a lactancia diseñado para este estudio, que permitió obtener antecedentes tanto de la madre como del niño e información sobre el acceso a lactancia materna, leche maternizada o mixta del niño/a durante el primer año de vida. Los datos de evaluación antropométrica durante el primer año de vida de los niños/as se obtuvieron a partir de los datos del Carnet de Control de Niño Sano, los cuales se registraron en una ficha creada para el estudio.

Ánalisis estadístico. Se reconocieron entre los datos, características de los niños del grupo estudio y control como: sexo, antecedente de lactancia materna y el tipo de FLMP para los niños con FLMP; usando valores absolutos y porcentajes. Se analizó si existían diferencias significativas usando Prueba de Chi Cuadrado (Amstalden-Mendes \& Gil-da-Silva-Lopes) o prueba exacta de Fisher, según correspondía.

Se construyeron gráficos de las trayectorias de ganancia de peso, talla y perímetro cefálico en el primer año de vida para ambos grupos segregados por sexo:

- Curva de peso para la edad.

- Curva de longitud para la edad.

- Curva perímetro cefálico para la edad

La desagregación por sexo es necesaria debido a que los patrones de crecimiento son diferentes en niños y niñas de acuerdo a las curvas de crecimiento OMS. (OMS 2006)

El análisis de la diferencia en peso, talla y perímetro cefálico en el nacimiento, 2, 4, 6, 8 y 12 meses de edad se realizó con la prueba $T$ de Student. Para todos los análisis se utilizó el programa Stata 11.0 (College Station, TX) StataCorp LP.) y se consideró un valor $p$ menor a 0,05 para considerar una diferencia como estadísticamente significativa.

\section{RESULTADOS}

De los 30 niños con FLMP, 4 (13,3\%) tenían FL, $10(33,3 \%)$ FP y 16 (53,4 \%) FLP. La lactancia materna exclusiva (LME) fue significativamente más frecuente en el grupo de niños sin FLMP $(73,3 \%)$ y ningún niño recibió únicamente leche de fórmula (LF). En niños con FLMP fue más frecuente la lactancia materna mixta (LMM) $(46,7 \%)$, seguido por uso exclusivo de LF $(33,3 \%)$ y LME (20\%). 
CORDERO E.; MADRID, P.; ESPINOZA, I.; ULLOA, C. \& PANTOJA R. Estudio comparativo de crecimiento estaturo ponderal y acceso a lactancia materna durante el primer año de vida de niños con fisura labio máxilo palatina versus niños sin fisura. Int. J. Odontostomat., 14(1):35-41, 2020.

En cuanto a la indicación de lactancia materna al momento del alta de maternidad en niños con FLMP fue mayoritariamente LMM (43,3\%) y en niños sin FLMP LME (83,3\%). Además un 16,67\% de las madres de niños con FLMP recibió indicación de administrar fórmula de manera exclusiva. Ninguna de las madres de niños sin FLMP recibió esta indicación.

El promedio de duración de la lactancia materna (libre demanda o programada) fue de 3 meses para los niños con FLMP y 10 meses para los niños sin FLMP. Ésta se extendió por seis meses o más tiempo en el 90 $\%$ de los niños sin FLMP comparado con un $20 \%$ en los niños con FLMP. El principal motivo, percibido por las madres de niños/as con FLMP, por el cual no pudieron instaurar o mantener la lactancia materna 6 meses o más fue la poca cantidad de leche $(62,5 \%)$, seguido por el mal incremento de peso del niño $(12,5 \%)$ y otros como el inicio de actividades laborales (8,2 \%), factores asociados a la FLMP (4,2\%), consejo del médico tratante $(4,2 \%)$, que el niño no quiso seguir amamantando $(4,2$ $\%)$ y falta de extractor de leche $(4,2 \%)$.

Al distribuir al grupo de niños/as con FLMP por tipo de fisura se observa que todos los niños/as a los que se les indicó LF o que tomaron exclusivamente
LF corresponden a niños/as con FLP o FP. Todos los niños con FL tomaron LME por 6 meses o más y utilizaron pecho y mamadera de manera conjunta como método de alimentación (Tabla I).

Crecimiento Estaturo-Ponderal. Las curvas de peso, longitud y perímetro cefálico de niños/as con FLMP muestran una desaceleración del crecimiento al segundo mes, en donde se observan las mayores diferencias dimensionales con el grupo de niños/as sin FLMP. Luego del sexto mes las curvas de niños con FLMP sugieren una recuperación, acercándose a las curvas del grupo control.

Peso. En el análisis de la trayectoria de curvas de peso en niños/as con y sin FLMP se estima que durante el primer año, un niño/a con FLMP pesa en promedio $450 \mathrm{~g}$ menos que un niño/a sin FLMP. La diferencia es mayor entre las niñas (600 g), que en niños (360 g).

Existen diferencias estadísticamente significativas de los promedios de peso durante el segundo, cuarto, sexto y octavo mes entre los niños/as con FLMP y sin FLMP. Al llegar al año, esta diferencia sigue siendo significativa sólo en las niñas, aunque su magnitud es menor que en meses anteriores (Fig. 1).

Tabla I. Duración y frecuencia lactancia materna en niños con FLMP, según clasificación anatómica.

\begin{tabular}{lccc}
\hline & $\begin{array}{c}\text { Fisura labial } \\
\mathrm{n}(\%)\end{array}$ & $\begin{array}{c}\text { Fisura palatina } \\
\mathrm{n}(\%)\end{array}$ & $\begin{array}{c}\text { Fisura labiopalatina } \\
\mathrm{n}(\%)\end{array}$ \\
\hline \multicolumn{1}{c}{ Duración lactancia materna** } & & & \\
6meses o más & $4(100,0)$ & $1(10,0)$ & $1(6,2)$ \\
Men os de 6 meses & -- & $4(40,0)$ & $10(62,6)$ \\
$\quad \begin{array}{l}\text { No recibió lactancia materna } \\
\quad \text { Frecuencia lactancia materna** }\end{array}$ & -- & $5(50,0)$ & $5(31,2)$ \\
$\quad$ & $4(100,0)$ & $2(20,0)$ & $2(12,5)$ \\
Libre demanda & -- & $3(30,0)$ & $9(56,3)$ \\
Programada & -- & $5(50,0)$ & $5(31,2)$ \\
No recibió lactancia materna & & &
\end{tabular}
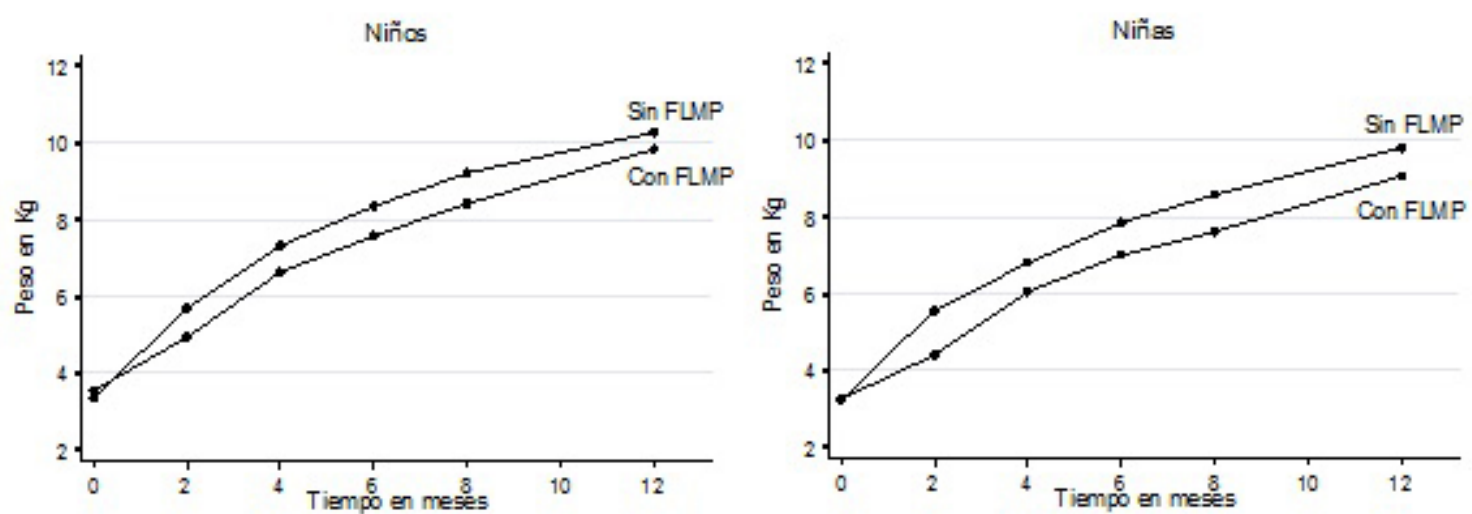

Fig. 1. Curva de peso para la edad durante el primer año de la de niñas y niños con y sin FLMP. 
En el análisis individual por niño se observó que, aunque la mayoría de éstos se encuentra en estado de eutrofia, hay más niños/as con FLMP que estuvieron en riesgo de desnutrición y bajo peso durante el primer año de vida, además todos los niños/as con bajo peso correspondían niños/as que presentaban fisura palatina, con o sin compromiso de labio (Tabla II).

Longitud. En el caso de las niñas con FLMP, aun cuando en promedio presentaron menor crecimiento que las niñas sin FLMP en toda la trayectoria $(0,94 \mathrm{~cm}$ menos que niñas sin FLMP); las diferencias sólo fueron estadísticamente significativas en el segundo mes. En los niños de ambos grupos, se encontraron diferencias significativas en la longitud durante el segundo, cuarto, sexto y duodécimo mes y se estima que niños con FLMP miden en promedio $1,11 \mathrm{~cm}$ menos que niños $\sin$ FLMP (Fig. 2)
La mayoría de los niños/as con FLMP tiene una talla normal, aunque dentro de la trayectoria de crecimiento se encuentran algunos niños/as de talla baja.

Perímetro cefálico. Si bien el promedio del perímetro cefálico de niños/as con FLMP es menor que el de los niños/as sin FLMP, no existen diferencias significativas entre ambos grupos (Fig. 3).

Todas las curvas se encuentran dentro de los parámetros de normalidad de la OMS. Sin embargo, a los dos meses, en la curva de peso para la edad, el promedio de niñas y niños con FLMP se encuentra al límite de -1DS indicando riesgo de desnutrición. El promedio de niños/as sin FLMP se encuentra sobres las curvas promedio OMS.

\begin{tabular}{|c|c|c|c|c|}
\hline & Tiempo & $\begin{array}{l}\text { Con FLMP Promedio } \\
(95 \% \text { IC) }\end{array}$ & $\begin{array}{c}\text { Sin FLMP Promedio } \\
(95 \% \text { IC) }\end{array}$ & $\begin{array}{l}\text { Diferencia } \\
(95 \% \text { IC) }\end{array}$ \\
\hline \multirow[t]{6}{*}{ Niñas } & Nacimiento & $3,26(2,98-3,55)$ & $3,24(3,02-3,46)$ & $0,03(-0,35-0,31)$ \\
\hline & 2 meses & $4,41(3,95-4,87)$ & $5,57(5,19-5,94)$ & $1,15(0,59-1,72)^{*}$ \\
\hline & 4 meses & $6,05(5,70-6,41)$ & $6,80(6,50-7,10)$ & $0,75(0,31-1,18)^{*}$ \\
\hline & 6 meses & $7,01(6,64-7,38)$ & $7,85(7,53-8,17)$ & $0,84(0,38-1,30)^{*}$ \\
\hline & 8 meses & $7,61(7,08-8,15)$ & $8,58(8,12-9,04)$ & $0,96(0,30-1,63)^{*}$ \\
\hline & 12 meses & $9,07(8,55-9,59)$ & $9,80(9,30-10,30)$ & $0,73(0,52-1,43)^{*}$ \\
\hline \multirow[t]{6}{*}{ Niños } & Nacimiento & $3,55(3,26-3,84)$ & $3,35(3,18-3,52)$ & $-0,20(-0,53-0,12)^{*}$ \\
\hline & 2 meses & $4,93(4,57-5,30)$ & $5,70(5,39-6,00)$ & $0,76(0,30-1,21)^{*}$ \\
\hline & 4 meses & $6,63(6,28-6,99)$ & $7,32(6,97-7,66)$ & $0,69(0,21-1,16)^{*}$ \\
\hline & 6 meses & $7,58(7,26-7,90)$ & $8,35(7,87-8,83)$ & $0,77(0,21-1,32)^{*}$ \\
\hline & 8 meses & $8,41(8,43-9,19)$ & $9,21(8,61-9,81)$ & $0,79(0,08-1,50)^{*}$ \\
\hline & 12 meses & $9,83(9,24-10,41)$ & $10,27(9,71-10,83)$ & $0,43(-0,34-1,22)$ \\
\hline
\end{tabular}

*T-Student $\mathrm{p}<0.05$
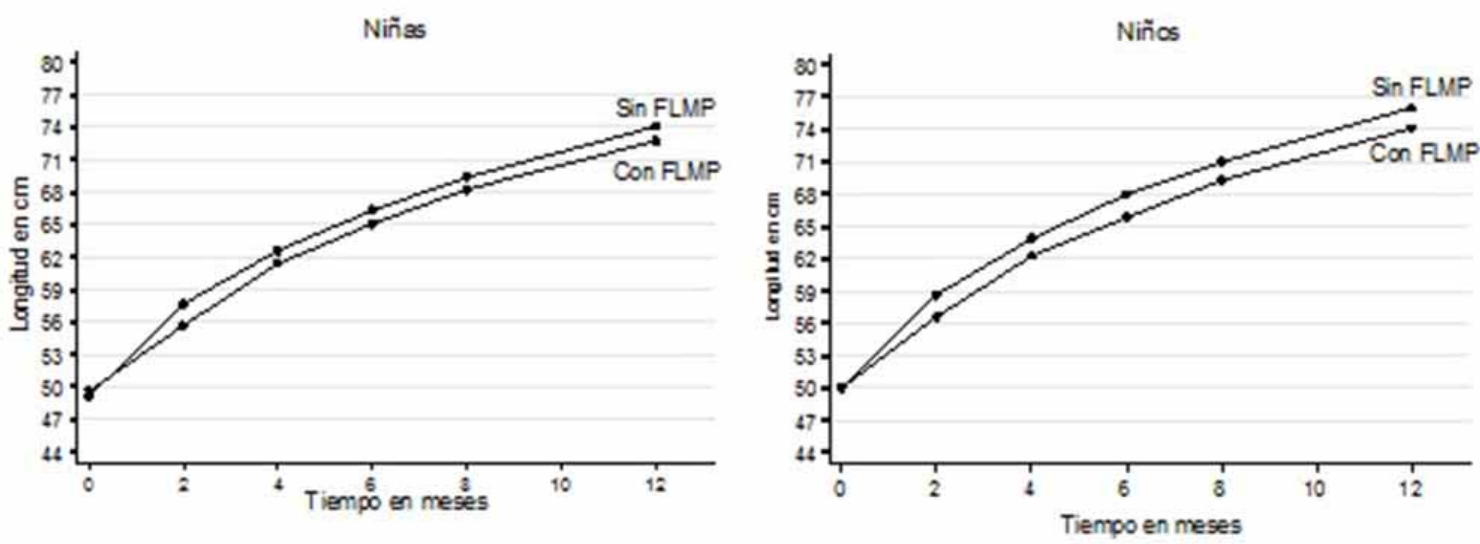

Fig. 2. Curva de Longitud para la edad durante el primer año de vida de niñas y niños con y sin FLMP. 

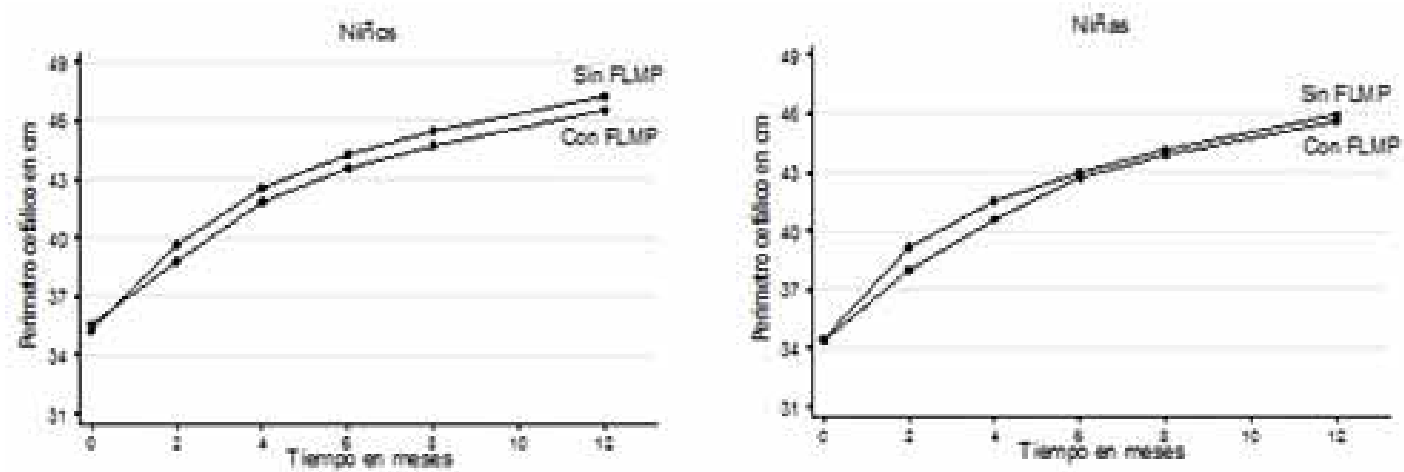

Fig. 3. Curva de perímetro cefálico para la edad durante el primer año de vida de niñas y niños con y sin FLMP.

\section{DISCUSIÓN}

Referente al acceso a lactancia materna, aunque ésta, en términos generales se fomentó en la mayoría de los casos, de forma mixta o exclusiva, $16,7 \%$ de las madres de niños con FLMP se les indicó alimentar a sus hijos sólo con LF, aún cuando en las recomendaciones de la guía clínica para el manejo de pacientes con FLMP se aconseja promover la lactancia materna de forma directa o con leche materna extraída (Ministerio de Salud).De acuerdo a las orientaciones OMS 2009, no existía ninguna razón médica aceptable para esta indicación, además se contradice con el estudio de Batista et al. (2011), que indica que fomentar la lactancia materna es un factor decisivo para su instauración y el no indicarla podría obedecer a falta de conocimiento de los profesionales o no reconocer la importancia de la misma.

Al hacer el análisis según clasificación anatómica de las FLMP, se observó que los niños con FL tuvieron éxito en la lactancia materna. La totalidad de éstos recibió LM exclusiva durante 6 meses o más, a libre demanda y utilizaron el pecho materno como método de alimentación, de forma combinada con mamadera, resultados que concuerdan con estudios que relacionan el tipo de fisura con la capacidad de los niños/as de generar una succión adecuada; se ha observado que niños con fisura labial, sin compromiso de paladar, son capaces de generar gradientes de presión intraoral similares a los niños sin FLMP sin afectar el proceso de lactancia materna (da Silva Dalben et al., 2003; Redford-Badwal et al., 2003; Montagnoli et al., 2005; Reid et al., 2007).

Pareciera ser que son las fisuras con compromiso de paladar las que tienen mayor interferencia en la lactancia materna, debido a que tienen afectado el pro- ceso succión al no lograr las gradientes de presión necesarias para un amamantamiento normal, lo que reafirma la importancia de realizar la intervención quirúrgica del velo del paladar a los 6 meses de edad o en cuanto las características de salud y peso del lactante lo permitan. Evaluando las prácticas de lactancia materna se observó que los niños/as con FLMP recibieron lactancia materna por menor tiempo y con menor frecuencia que los niños sin fisura, lo que es concordante con la literatura investigada (Smedegaard et al., 2008; Campillay et al., 2010).

Evaluar el estado psicológico de las madres no era un objetivo de este trabajo, pero es un factor relevante que afecta al vínculo que éstas puedan lograr con sus hijos con FLMP, influyendo directamente en las prácticas de lactancia materna, por lo que sería interesante incluirlo en investigaciones futuras. El diagnóstico prenatal de la FLMP, puede contribuir al ajuste postparto materno positivo y no tenerlo constituye un factor de riesgo para la depresión post-parto (Johns et al., 2018).

En cuanto a factores sociodemográficos de la madre el único que muestra una diferencia entre ambos grupos es el nivel de escolaridad. Se observó que hay un mayor porcentaje de madres de niños sin fisura con educación superior, técnica o universitaria, esto es un factor predisponente positivo para lactancia materna, pues se relaciona con mayores conocimientos y comprensión sobre los beneficios que tiene la leche materna en la salud de sus hijos. En las madres de niños con FLMP vemos una mayor cantidad de madres con educación básica - concordante con el estudio de Martínez Urbina (2017), las cuales no dieron lactancia materna o lo hicieron por menos de 6 meses. 
CORDERO E.; MADRID, P.; ESPINOZA, I.; ULLOA, C. \& PANTOJA R. Estudio comparativo de crecimiento estaturo ponderal y acceso a lactancia materna durante el primer año de vida de niños con fisura labio máxilo palatina versus niños sin fisura. Int. J. Odontostomat., 14(1):35-41, 2020

Los niños con FLMP de ambos sexos en este estudio presentaron dimensiones corporales más pequeñas en relación al grupo control. El peso fue el parámetro más comprometido para ambos sexos. Si bien niños y niñas con y sin FLMP nacieron en promedio con pesos similares, a los dos meses se observa la mayor diferencia de peso entre el grupo estudio y control, segregados por sexo. A los dos meses existió una mayor cantidad de niños/as con FLMP con bajo peso o riesgo de desnutrición que en toda la trayectoria desde el nacimiento hasta el año de vida, bajando el promedio de peso en ese mes en el grupo estudio lo que podría explicar esta mayor diferencia. Sin embargo, al observar las curvas de cada niño, a los dos meses se observa una menor ganancia de peso generalizada en el grupo de niños/as con FLMP, respecto a los niños/as sin FLMP; Es posible que esta menor ganancia de peso durante los primeros meses, sea consecuencia de la adaptación del bebé a los métodos de nutrición.

Aun cuando los niños/as con FLMP presentan menor peso promedio que los niños/as sin FLMP durante el primer año de vida, la curva promedio sugiere una "recuperación" posterior a los 6 meses.

Si bien, en ningún momento las curvas del grupo estudio y control se superponen luego del nacimiento, la curva de los niños/as con FLMP comienza a acercarse a la curva de los niños/as sin FLMP, en especial en el género masculino. Incluso, al compararlas con las referencias OMS, las curvas de niños/as con FLMP que se encontraban bajo la media OMS, luego de los 6 meses comienzan a acercarse, hasta superarla por completo al año de vida.

Los niños/as con bajo peso FP o FLP; las dificultades nutricionales pueden haber comprometido el crecimiento, ya que el parámetro más afectado fue el peso y es el más susceptible a una nutrición inadecuada. Como ya se mencionó los niños/as con FP y FLP tienen más problemas al momento de alimentarse al tener alterado el proceso de succión.

El segundo parámetro más afectado fue la longitud. Para ambos sexos, el grupo estudio mostró una desaceleración del crecimiento a los dos meses, coincidiendo con el mes de menor ganancia de peso. Estos parámetros están fuertemente relacionados entre sí, por lo que la menor longitud promedio al segundo mes podría ser explicado por esto. En el caso particular del género masculino, estos presentaron diferencias significativas en el promedio de longitud durante casi toda la trayectoria desde el nacimiento hasta el primer año de vida; esto pudo ocurrir por la presencia de niños con talla alta o en el límite de ésta en los niños del grupo control, aumentando considerablemente el promedio de longitud de niños sin FLMP. Además, se ha reportado en la literatura una asociación entre la deficiencia de hormona de crecimiento y la presencia de FLMP, afectando en la estatura de estos niños/as, sin embargo esto debe estudiarse en cada individuo luego de descartar factores nutricionales que estén afectando en el crecimiento (Abdollahi-Fakhim et al., 2015 ).

En cuanto al perímetro cefálico, si bien no existieron diferencias estadísticamente significativas entre ambos grupos segregados por sexo; en la curva de circunferencia craneal promedio de niños y niñas con FLMP se observa una desaceleración del crecimiento del perímetro cefálico a los dos meses, al igual que en los dos parámetros anteriores.

Debido a los resultados dispares que presentaron los niños/as con FLMP respecto a la lactancia materna entre los niños con fisura labial aislada y niños/as cuyas fisuras comprometían el paladar, como sugerencia para la investigación científica futura sería interesante correlacionar cada tipo de fisura con la evolución de la alimentación y los parámetros de crecimiento. En este caso la muestra niños con fisura labial aislada fue muy pequeña para realizar generalizaciones significativas.

\section{CONCLUSIONES}

1. Los niños/as con FLMP reciben menos lactancia materna y tienen un menor crecimiento estaturo ponderal que los niños/sin FLMP durante el primer año de vida, siendo crítico a los dos meses de edad y recuperándose luego de los 6 meses, coincidiendo con el período en que se realiza la cirugía primaria según la cronología funcional de Delaire, utilizada en el HCSBA.

2. El tipo de fisura más afectado son las fisuras de paladar - con o sin compromiso de labio - reafirmando la importancia de la intervención quirúrgica del velo del paladar a edades tempranas.

3. Los niños con fisura tienen el mismo potencial de crecimiento y desarrollo que el resto de los niños sin patologías asociadas. Alcanzar dicho potencial dependerá de la identificación, del conocimiento y de la modificación de diversos factores ambientales que afecten la alimentación del lactante; entre ellos la correcta y oportuna orientación por parte de los profesionales de la salud 
sobre la alimentación y los métodos de lactancia, teniendo un papel fundamental en la instauración y mantención de la lactancia materna.

4. Existen falencias en el asesoramiento a los padres por parte del equipo de salud en niños/as fisurados, por lo que es necesario incentivar y capacitar a los profesionales a fomentar la lactancia materna en madres de niños/as con FLMP.

AGRADECIMIENTOS. Nuestros pacientes y su familia, personal técnico de Servicio de Cirugía Maxilo facial HSBA y Centro de Salud Familiar Las Mercedes.

CORDERO E.; MADRID, P.; ESPINOZA, I.; ULLOA, C. \& PANTOJA, R. Comparative growth and development study in children with cleft lip and palate fissure versus children without fissure. Int. J. Odontostomat., 14(1):35-41, 2020.

ABSTRACT: The maxillary cleft lip and palate fissure (CLPF) is a public health problem in Chile. It involves the lack of fusion of the lip and / or the palatal processes, generating a skeletal muscle imbalance that prevents normal facial development, further affecting vital functions such as breathing, sucking and swallowing. It may also cause nutritional problems. An observational, analytical, retrospective cohort study was carried out, to assess the growth in weight status of breastfeeding infants with maxillary CLPF, and compare it with children without fissure during the first year of life. The study was based on data obtained in 2017, from interviews with mothers of infants with non-syndromic CLPF, and those without CLPF from the same region in Chile. In children without CLPF, exclusive breastfeeding predominated, while children with CLPF received mixed breastfeeding (47.7\%) and exclusive use of formula milk (33.3 $\%)$. During the first year of life, infants with CLPF weighed an average of $0.45 \mathrm{~kg}$ and measured $1.11 \mathrm{~cm}$ less, than children without fissures. Children with CLPF received less breastfeeding and ranked lower in weight and growth than children / without CLPF during the same time period, with weight being the most affected growth parameter. and height.

KEY WORDS: cleft lip palate, breastfeeding, weight

\section{REFERENCIAS BIBLIOGRÁFICAS}

Abdollahi-Fakhim, S.; Bayazian, G.; Shiva, S.; Sohrabpour, M. \& Ebrahimzade, Y. Growth hormone deficiency in cleft lip and palate patients. J. Anal. Res. Clin. Med., 3(4):231-5, 2015.

Amstalden-Mendes, L. G. \& Gil-da-Silva-Lopes, V. L. Fenda de lábio e ou palato: recursos para alimentação antes da correção cirúrgica. Rev. Cienc. Med. (Campinas), 15(5):437-48, 2006.

Batista, L. R. V.; Triches, T. C. \& Moreira, E. A. M. Desenvolvimento bucal e aleitamento materno em crianças com fissura labiopalatal. Rev. Paul. Pediatr., 29(4):674-9, 2011.
Campillay, P. L.; Delgado, S. E. \& Brescovici, S. M. Avaliação da alimentação em crianças com fissura de lábio e/ou palato atendidas em um hospital de Porto Alegre. Rev. CEFAC, 12(2):257-66, 2010.

Cauvi, D. \& Leiva, N. Etiopatogenia y Tratamiento de las Fisuras Labio-Máxilo-Palatinas. Santiago de Chile, Facultad de Odontología, Universidad de Chile, 2004.

Cortés Araya, J.; Niño Duarte, A. Y.; Sung Hsieh, H. H. \& Cortés Cuadra, P. Estrategia terapéutica en las fisuras labio-máxilo-palatinas. La aproximación funcional de Delaire. Rev. Esp. Cir. Oral Maxilofac., 24(1):13-9, 2002.

da Silva Dalben, G.; Costa, B.; Gomide, M. R. \& Teixeira das Neves, L. T. Breast-feeding and sugar intake in babies with cleft lip and palate. Cleft Palate Craniofac. J., 40(1):84-7, 2003.

Gil-da-Silva-Lopes, V. L.; Xavier, A. C.; Klein-Antunes, D.; Ferreira, A. C.; Tonocchi, R.; Fett-Conte, A. C.; Silva, R. N.; Leirião, V. H.; Caramori, L. P.; Magna, L. A.; et al. Feeding infants with cleft lip and/or palate in Brazil: suggestions to improve health policy and research. Cleft Palate Craniofac. J., 50(5):577-90, 2013.

Johns, A. L.; Hershfield, J. A.; Seifu, N. M. \& Haynes, K. A. Postpartum depression in mothers of infants with cleft lip and/or palate. $J$. Craniofac. Surg., 29(4):e354-e358, 2018.

Markus, A. F.; Delaire, J. \& Smith, W. P. Facial balance in cleft lip and palate. I. Normal development and cleft palate. Br. J. Oral Maxillofac. Surg., 30(5):287-95, 1992.

Martínez Urbina, G. K. Perfil epidemiológico de los pacientes con fisuras labio-máxilo-palatina no sindrómicas atendidos en el Hospital San Borja Arriarán durante el período 2005-2015. Tesis de Pregrado. Santiago de Chile, Facultad de Odontología, Universidad de Chile. 2017.

Ministerio de Salud. Guía Clínica AUGE Fisura Labiopalatina. Santiago de Chile, Subsecretaría de Salud Pública, División de Prevención y Control de Enfermedades, Departamento de Salud Bucal, Ministerio de Salud, Gobierno de Chile, 2015. Disponible en: http:/ /www.bibliotecaminsal.cl/wp/wpcontent/uploads/2016/04/ guiaFisuraLabioPalatina-2015-CM.pdf

Montagnoli, L. C.; Barbieri, M. A.; Bettiol, H.; Marques, I. L. \& de Souza, L. Growth impairment of children with different types of lip and palate clefts in the first 2 years of life: a cross-sectional study. J. Pediatr. (Rio J), 81(6):461-5, 2005.

Redford-Badwal, D. A.; Mabry, K. \& Frassinelli, J. D. Impact of cleft lip and/or palate on nutritional health and oral-motor development. Dent. Clin. North Am., 47(2):305-17, 2003.

Reid, J. A review of feeding interventions for infants with cleft palate. Cleft Palate Craniofac. J., 41(3):268-78, 2004.

Reid, J.; Reilly, S. \& Kilpatrick, N. Sucking performance of babies with cleft conditions. Cleft Palate Craniofac. J., 44(3):312-20, 2007.

Smedegaard, L.; Marxen, D. R.; Moes, J.; Glassou, E. N. \& Scientsan, C. Hospitalization, breast-milk feeding, and growth in infants with cleft palate and cleft lip and palate born in Denmark. Cleft Palate Craniofac. J., 45(6):628-32, 2008.

\section{Dirección para correspondencia:}

Erita Cordero Carrasco

Facultad de Odontología

Universidad de Chile

Sergio Livingstone Pohlhammer 943

Independencia

Santiago

CHILE

Email: eritac@uchile.cl

Recibido : 09-07-2019

Aceptado: 12-08-2019 